\title{
Observer strategies in dichoptic viewing of successive stimuli
}

\author{
DONALD H. THOR, E. R. JOHNSTONE TRAINING AND \\ RESEARCH CENTER, Bordentown, New Jersey
}

Enhanced ability to correctly perceive order of brief visual stimuli presented dichoptically may be interpreted as learning by the observer to adopt an appropriate strategy.

An $S$ can be made to view successively presented visual stimuli "dichoptically" by using polarizing filters oriented over the stimuli and at the eyepiece so that a different stimulus is seen by each eye at the same locus of the visual field. Using a three channel tachistoscope to obtain a pre-adapting field and a dark interstimulus field as well as fields for the two different stimuli (a square and a triangle), Robinson (1967) presented 10 msec stimuli separated by a variable ISI (interstimulus interval). His Ss were reported capable of determining correct order when only $5 \mathrm{msec}$ separated the two stimuli. A replication of his study (Thor, 1967) was not successful in duplicating his finding of heightened discriminative capacity of the human observer when stimuli were viewed dichoptically.

A careful consideration was made of the possible cues involved in the performance of one observer in the replication study who was able to correctly discriminate the order of two visual stimuli of $10 \mathrm{msec}$ duration with zero interstimulus interval. This $\mathrm{S}$ was found to be reacting to one or both of two cues in the operation of the control panel of the Scientific Prototype three channel tachistoscope. When the fixation field is not turned off during switching of the function control knob (to change order of presentation) there is a visual cue consisting of a very slight and fast blink in luminance of the fixation field. The function control knob simultaneously yields an auditory cue indicating a change in order to the observer who is aware of the contingency. Awareness of the change in the order of presentation is apparently sufficient to allow errorless performance. When these two cues were masked, the ability of this outstanding observer fell to the level of other Ss who were unaware of these cues.

Method. To test Robinson's claim that several hundred trials over a number of sessions are required for proficiency in dichoptic viewing of successive stimuli, a graduate student was selected who was naive regarding apparatus or intent of the study and had no previous experience in viewing tachistoscopically presented stimuli. This $\mathrm{S}$ received 72 trials at each daily session over a period of two weeks according to the procedure previously described (Thor, 1967), with 12 trials at each of six interstimuli durations and with a $10 \mathrm{msec}$ exposure duration of Stimulus 1 and also of Stimulus 2 . The visual cue was controlled by turning off the red fixation field during each switching of the function selector. However, the audition cue concomitant with the audible switching of the function selector was allowed to remain.

Instructions were similar to those used previously but a strong implication was made that it was possible to learn to perform the task with great accuracy if the observer were astute. No feedback of accuracy was given after individual trials, but after sessions the $\mathrm{S}$ was informed of general progress (e.g., "you were correct more often today").

Results. During the first five consecutive sessions (see Fig. 1), the error rate dropped to a low of three errors on the fifth session, all occurring at zero interstimulus interval. On the sixth session earphones (Lafayette Model F-767) were placed on the observer and he was subjected to white noise $(75 \mathrm{~dB}$ at $3 \mathrm{~cm}$ from each earphone as measured with a Bruel and Kjaer Type 2203 sound meter) to mask sound made by the function control knob. The anticipated increase in error did not occur; in fact, there was a drop from three to one error in 72 trials. These results suggested that this $S$ was using some cue other than the visual or auditory cues referred to previously. A cue was indicated since the cumulative curves show what may be considered a learning process occur- ring over a number of sessions and hundreds of trials. After six sessions and 432 trials it is clear that performance was nearly asymptotic. The reduction of errors over blocks of trials at the zero ISI may be interpreted as learning to discriminate succession with no time between stimulus presentations.

When questioned, the $S$ described his strategy by referring to the manner of aiming a rifle when shooting skeet. Visual accommodation was voluntarily altered so that the images of triangle and square subjectively appeared in slightly different locations in visual space when presented in rapid succession: with images phenomenally in lateral proximity the $S$ attended to apparent movement, and the direction of apparent movement served as the cue to order of presentation. With movement appearing to the left, the triangle was reported as appearing first. With movement to the right, the square was reported as seen first.

Discussion. The use of apparent motion as an accurate cue has been tested by teaching naive Ss to perform comparably to highly experienced $\mathrm{S}$ s in only one viewing session. Accurate discriminations of order can be obtained in relatively few trials with ISIs of only 5 or $10 \mathrm{msec}$. Further tests without the polarizing filters have revealed that comparable accuracy can also be obtained with normal binocular viewing. The presence of apparent movement (for review see Graham, 1965) at time intervals under consideration has been noted by Hirsch \& Sherrick (1961) who have also speculated upon the possibility of apparent motion as a cue for successivity determination.

A more general problem illustrated by the present example of observer strategies is the degree to which such behavior may play a role in similar research designs. The present account demonstrates the ability of observer strategies to function when stimulus presentations are of a few msec duration. A unique aspect of the use of apparent motion is that the cue itself is not external to the observer and thus presents an unusual control problem.

Robinson's finding of the necessity for Ss to acquire controlled fixation over several weeks of practice to demonstrate heightened observer capability when viewing successive stimuli presented dichoptically is corroborated by the present data. Conclusions regarding the influence of dichoptic viewing upon the perceptual capability of the central visual mechanism remain in question since effective cues have not been eliminated. Gross differences in observer discriminative capability with increasing familiarity of the experimental situation may be more parsimoniously attributed to learning to use appropriate cues than to neuro-physiological processes.

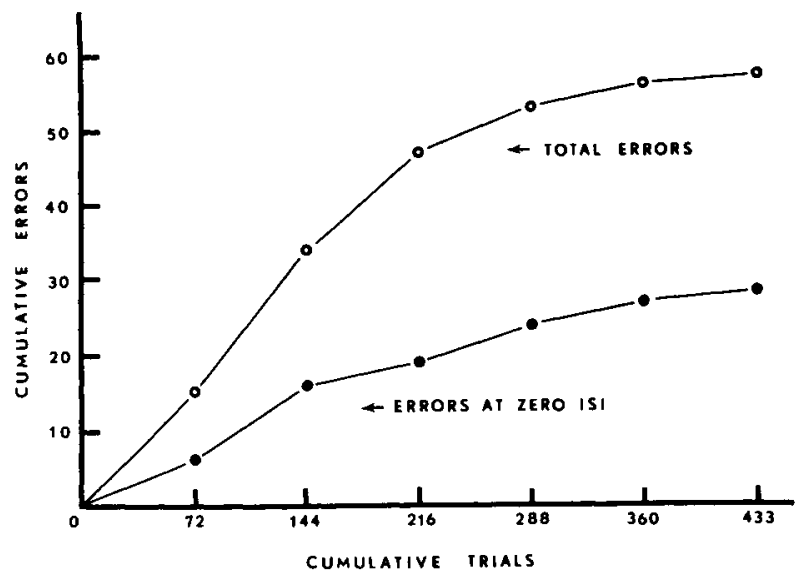

Fig. 1. Change in error rate for one $S$ over successive sessions of 72 trials. An error was an incorrect call of the order of two successive stimuli of 10 msec duration. Interstimulus durations were $0,20,40,60,80$, or $100 \mathrm{msec}$. 
REFERENCES

GRAHAM, C. H. Perception of movement. In C. H. Graham (Ed.), Vision and visual perception. New York: Wiley, 1965, 580-583.

HIRSCH, 1., \& SHERRICK, C. Perceived order in different sense modalities. J. exp. Psychol, 1961, 62, 423-432.

ROBINSON, D. N. Visual discrimination of temporal order. Science, 1967, $156,1263-1264$

THOR, D. H. Dichoptic viewing and temporal discrimination: An attempted replication. Science, 1967, 158, 1704-1705.
NOTES

1. Robinson (1967) has incorrectly referred to Hirsch and Sherrick (1961) as employing the monocular viewing condition. In fact, Hirsch and Sherrick used binocular viewing (personal communication from C. Sherrick).

2. I thank John Winters and William Emener who served as Ss and offered helpful suggestions, David Hoats for assistance with apparatus, and other members of the Johnstone research staff for their critical reading of the manuscript. 